\title{
Viabilidad y germinación de semillas de Puya raimondii Harms (Bromeliaceae)
}

\author{
Viability and germination of seeds of Puya raimondii Harms (Bromeliaceae)
}

Giovana Vadillo ${ }^{1}$, Mery Suni ${ }^{1}$ y Asunción Cano ${ }^{2}$

Presentado: $28 / 10 / 2003$

Aceptado: $09 / 07 / 2004$

\section{Resumen}

Puya raimondii Harms es una especie endémica de la zona altoandina del Perú y Bolivia, distribuida entre 3200 y $4800 \mathrm{~m}$ de altitud que sobresale por su gran tamaño y belleza. Es considerada una especie amenazada, por ello la importancia de conocer los factores que afectan la viabilidad y germinación de sus semillas, su único medio de propagación natural. Se colectaron semillas de $P$. raimondii en 1999 y 2000 de los rodales de Huashta Cruz (distrito Pueblo Libre, departamento Ancash, Perú) y se clasificaron en lotes según sus características externas. Se les determinó la viabilidad (calidad) por el método de tetrazolium y el contenido de humedad. En una selección de 5 lotes se evaluaron el efecto de la luz y la temperatura en la germinación. Se encontró que la viabilidad de las semillas depende de las condiciones a que estuvieron expuestas in situ manifestados por las características externas de las semillas y que se correlaciona con su contenido de humedad, afectando inclusive su apariencia. Las semillas expuestas a la luz presentaron alto poder germinativo (fotoblásticas positivas, requieren luz para germinar). Se encontró que temperaturas mayores a $21^{\circ} \mathrm{C}$ afectan negativamente el porcentaje de germinación y el índice de velocidad de germinación.

Palabras claves: Puya raimondii, Bromeliaceae, germinación, viabilidad de semilla.

\section{Abstract}

Puya raimondii Harms is an endemic species of the highlands of Peru and Bolivia between 3200 to $4800 \mathrm{~m}$ of altitude with noticeable great size and beauty. It is considered threatened species; therefore it is urgent to know the factors that affect its viability and germination of their seeds; aspects focused work presently. The seeds were collected in 1999 and 2000 in Huashta Cruz (Pueblo Libre, Ancash, Peru) and they classified as lots (considering their origin and appearance). They were determined their moisture content and the viability (quality). By means of germination tests, it was evaluated in 5 lots the effect of the light and temperature. The seeds presented different viability which depended on the conditions they were exposed in situ and that it is correlated inversely with its content of humidity, affecting its appearance inclusive. The seeds exposed to the light presented high percentage of germination (positive photoblastics, require light to germinate). Temperatures higher than $21^{\circ} \mathrm{C}$ had a negative effect in the percentage of germination and germination speed index.

Key words: Puya raimondii, Bromeliaceae, germination, seed viability,

\section{Introducción}

Puya raimondii Harms (Bromeliaceae) es una especie distribuida en áreas restringidas de los Andes de Perú como rodales entre 3300$4300 \mathrm{~m}$ de altitud llegando hasta Bolivia. Esta

${ }^{1}$ Laboratorio de Fisiología Vegetal. Facultad de

Ciencias Biológicas. Universidad Nacional Mayor de San Marcos. Lima. Perú.

Email Giovana Vadillo: gpvadillo@hotmail.com, Email Mery Suni: msunin@unmsm.edu.pe

${ }^{2}$ Museo de Historia Natural, Universidad Nacional Mayor de San Marcos. Lima. Perú.

Email Asuncion Cano: acanoe@unmsm.edu.pe especie puede vivir en forma vegetativa por más de 40 años antes que se inicie la floración, después de la cual la planta muere (Rivera, 1985). Al florecer, la planta produce (potencialmente) entre 6 a 12 millones de semillas (Rivera, 1985 y Cano et al., 2000) pese a ello su población y área de distribución han ido disminuyendo; debido probablemente a que su población está amenazada por factores naturales (herbívoros, plagas y enfermedades) y humanos (quema y tala), de allí que se esta- 
blezcan preferentemente en lugares rocosos y con pendientes los que le protegerían del pastoreo y fuertes vientos (Rivera, 1985). Actualmente Puya raimondii es considerada una especie amenazada y está protegida desde 1973 por el Decreto Supremo 1014 del Ministerio de Agricultura (Rivera, 1985; Venero \& Hostning, 1986).

Se conoce muy poco sobre los factores que afectan la viabilidad y germinación de las semillas de $P$. raimondii. Han sido publicadas referencias generales, hasta contradictorias, donde no se precisa las condiciones en que fueron evaluadas las semillas. Por ejemplo, Hartmann (1981) encuentra un 90\% de germinación a los 32 días, manteniendo su poder germinativo por seis meses, mientras que Rivera (1985) menciona un 99\% a los 18 días y que mantiene la viabilidad por años. Además Smith y Downs (1974) hacen notar el importante papel que cumple la temperatura en la germinación de Puya, al mencionar que $P$. berteroniana tiene un buen porcentaje de germinación a $15^{\circ} \mathrm{C}$ y que va disminuyendo a medida que la temperatura aumenta hasta $\operatorname{los} 25^{\circ} \mathrm{C}$. Así mismo ensayos preliminares realizados por Suni et al. (2001) indican que la temperatura baja podría actuar como un factor condicionante para la posterior germinación y que la luz sería un factor importante.

El presente estudio tiene como objetivo determinar la viabilidad y los factores que afectan la germinación de las semillas de Puya raimondii, lo cual permitirá conocer aspectos importantes de su propagación natural. De esta manera se contribuirá al conocimiento de la dinámica poblacional de esta especie y a su conservación.

\section{Materiales y métodos}

Se trabajó con semillas de los rodales de Huashta Cruz (distrito Pueblo Libre, provincia Huaylas, departamento Ancash) colectadas en 1999 y 2000 . Las semillas fueron agrupadas en 56 lotes (Anexo 1) de acuerdo a la fecha de colecta, características externas de coloración (clara y oscura), estado aparente (buenas, quemadas, semiquemadas y muy quemadas) y temperatura de almacenamiento (medio ambiente con $21{ }^{\circ} \mathrm{C}$ aproximadamente o en frío con $11^{\circ} \mathrm{C}$ ).

Se escogieron los lotes que tenían semillas con características de blancas o oscuras y buenas o mixtas los que totalizan 22 lotes. En los 22 lotes se evaluó la viabilidad (calidad) con el método de Tetrazolium (TZ). Se consideró semilla viable aquella cuyo embrión se teñía de rojo (Fig. 1).

Cinco lotes con resultados positivos fueron seleccionados para las pruebas de germinación (lotes 15, 28, 34, 41 y 50). Estos fueron caracterizados mediante la prueba de viabilidad y medida del contenido de humedad de las semillas. El contenido de humedad de las semillas en peso seco (\% Hps) se determinó secando en la estufa a $120^{\circ} \mathrm{C}$ por $24 \mathrm{o}$ 48 horas, 4 repeticiones de $0,1 \mathrm{~g}$ c/u por lote. E1 21/3/01 y el 26/6/01 se realizaron dos evaluaciones del contenido de humedad de las semillas antes de ser colocados en bolsas herméticas. La prueba de viabilidad realizada por el método de tetrazolium en dos fechas la primera fecha, el 15/03/01, cuando estaban en bolsas con cierre al inicio del experimento y la segunda fecha, el 25/09/01, tres meses después de haber sido colocadas en bolsas herméticas de aluminio biplastificadas.

Las pruebas de germinación consideraron el efecto de la luz y temperatura en la germinación. Los tratamientos aplicados se muestran en la Tabla 1. Los tratamientos de oscuridad se realizaron cubriendo totalmente las placas con papel aluminio y evaluadas semanalmente con luz verde de seguridad.

Para realizar las pruebas de germinación se utilizó $0,2 \mathrm{~g}$ (equivalente a 400 semillas) de cada lote por cada tratamiento evaluado ( 5 Repeticiones). Se desinfectó con hipoclorito de sodio al $2,6 \%$ por 30 minutos y se enjuagó 6 veces con agua destilada estéril. Se sembró en la cámara de flujo laminar de 50-70 semi- 


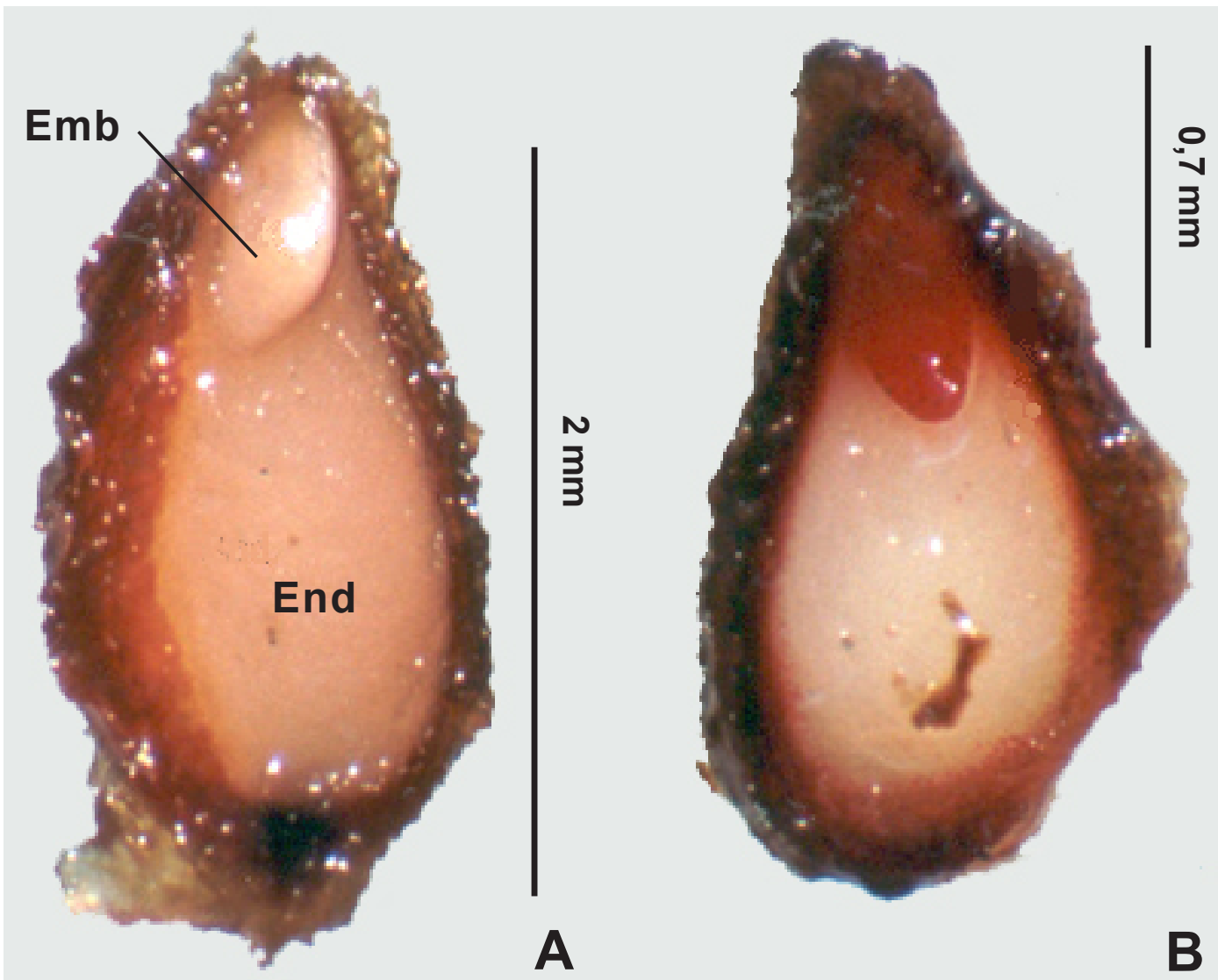

Figura 1. Evaluación de la viabilidad de las semillas de Puya raimondii con el método Tetrazolium. A, semilla con embrión no teñido (no viable); B, semilla con embrión teñido (viable). Embrión (Emb), endospermo (End).

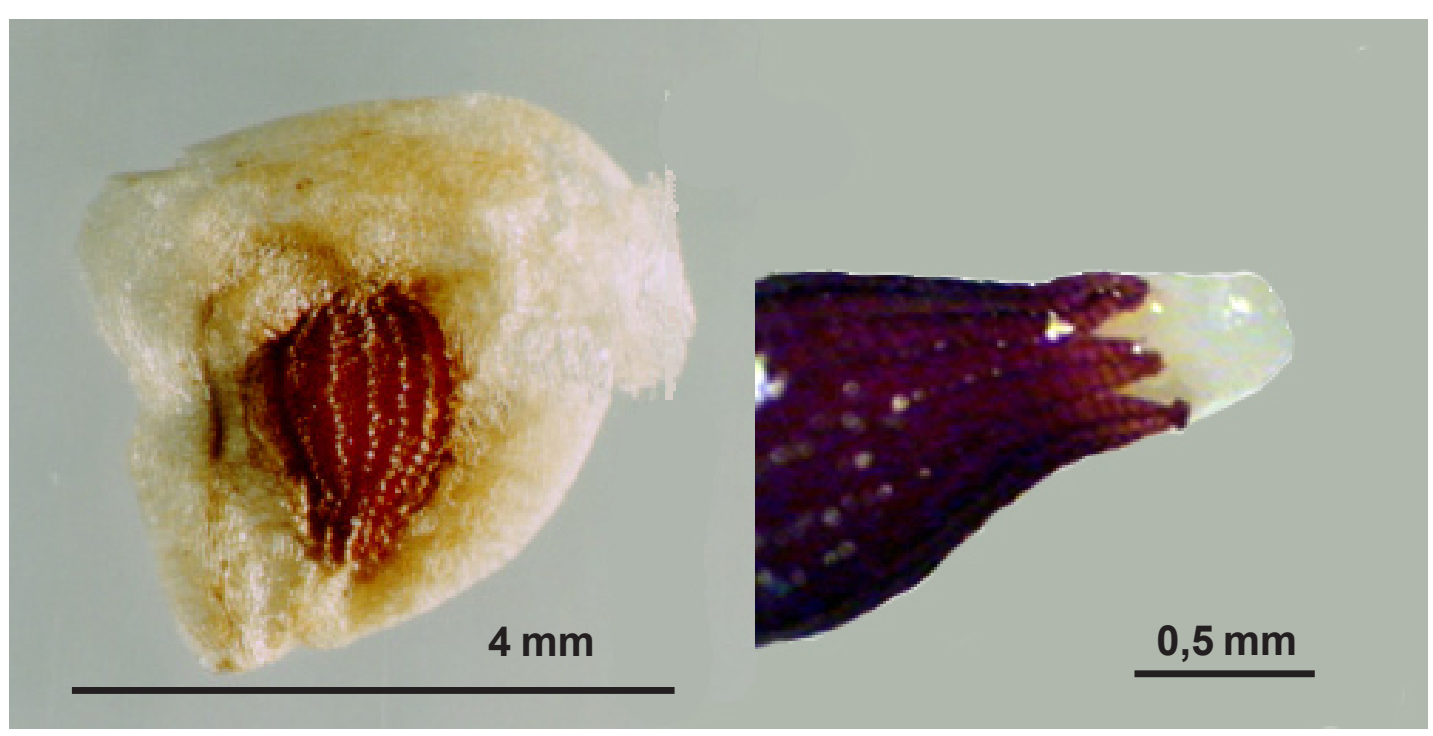

Figura 2. Semilla de $P$. raimondii (izquierda), semilla germinada (derecha). 
Tabla 1. Tratamientos en las pruebas de germinación. CN: condiciones naturales. CC: condiciones controladas. Factor aplicado:(+) y no aplicado: (-).

\begin{tabular}{|c|c|c|c|c|}
\hline Tratamiento & Luz Natural & 9 horas luz & Oscuridad & Temperatura \\
\hline \multicolumn{5}{|c|}{ Primera prueba de germinación 19/06/2001 } \\
\hline CN 1 & + & - & - & $\left(18,5-23^{\circ} \mathrm{C}\right)$ \\
\hline $\mathrm{CN} 2$ & - & - & + & $\left(18,5-23^{\circ} \mathrm{C}\right)$ \\
\hline $\mathrm{CC} 3$ & - & + & - & $18^{\circ} \mathrm{C}$ \\
\hline CC 4 & - & - & + & $18^{\circ} \mathrm{C}$ \\
\hline \multicolumn{5}{|c|}{ Segunda prueba de germinación 19/10/2001 } \\
\hline $\mathrm{CN} 5$ & + & - & - & $\left(21-26,5^{\circ} \mathrm{C}\right)$ \\
\hline
\end{tabular}

1las por placa (5 placas por tratamiento) y luego se las rotuló y selló. La distribución de las placas en el área de cultivo fue al azar. Las evaluaciones se realizaron dos veces por semana, retirando las semillas germinadas. Se consideró germinada cuando la radícula había emergido 0,5 mm aproximadamente (Fig. 2). Con los datos obtenidos se calculó el porcentaje de germinación $(\% \mathrm{G})$ y el Índice de velocidad de germinación (IVG), donde:

$$
\text { IVG }=\sum\left(n_{i} / t_{i}\right)
$$

$\mathrm{n}_{\mathrm{i}}=$ Número de semillas germinadas en el i-ésimo día;

$t_{i}=$ Tiempo en días, para la germinación en el i-ésimo día;

Para el procedimiento estadístico los datos fueron transformados a Arcsen $\sqrt{(\mathrm{G} \% / 100)}$ para la aproximación a una curva normal (Steel \& Torrie, 1980).

\section{Resultados}

\section{Evaluación de la viabilidad (calidad) de las semillas}

El promedio general de la viabilidad de los 22 lotes seleccionados, fue de $41,85 \%$. Además se puede observar en la figura 3 y Anexo 1 que las semillas de los lotes clasificados como claras y buenas tienen los más altos valores de viabilidad. Los resultados de viabilidad permitieron clasificarlos en muy buenas $(>70 \%)$, buenas (50 a $70 \%)$, regulares $(30$ a $50 \%)$ y malas (<30\%) (Fig. 3).

\section{Caracterización y evaluación de los cin- co lotes seleccionados}

\section{Viabilidad}

Al evaluar la viabilidad se obtuvo diferencias significativas entre los lotes en la segunda fecha, donde el lote 50 tuvo menor viabilidad (Tabla 2). Haciendo el análisis por lote

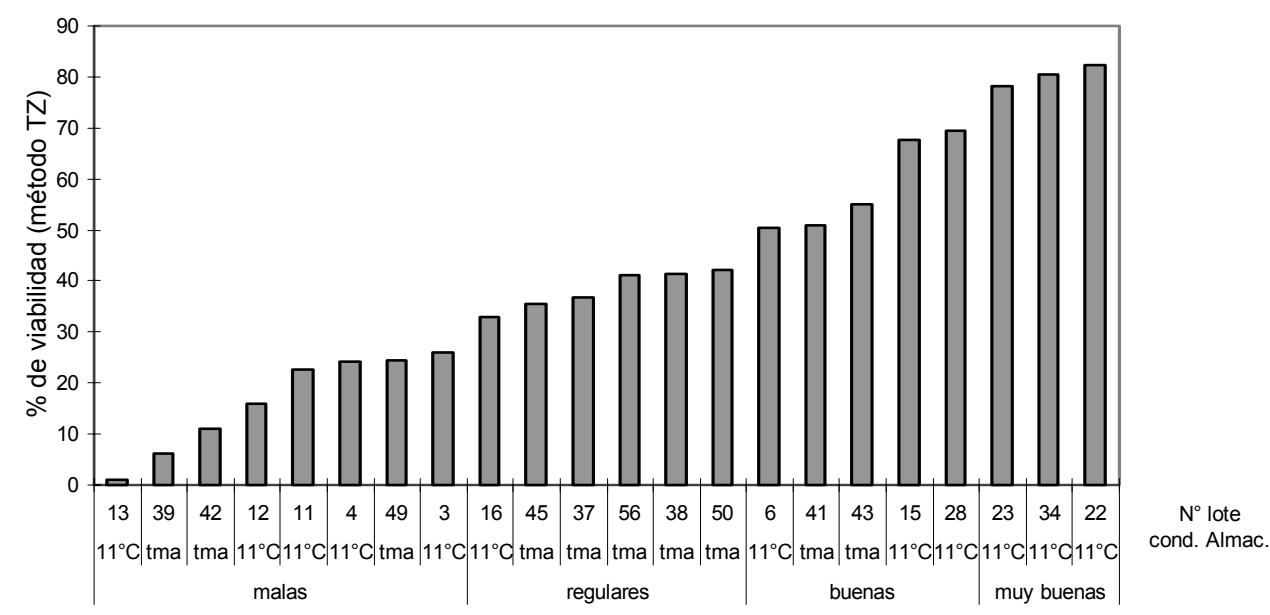

Figura 3. Clasificación de los lotes según su Viabilidad (método tetrazolium), clasificadas como muy buenas, buenas, regulares y malas ( $>70$, de 50 a 70 , de 30 a 50 y $<30 \%$ respectivamente). tma $=$ temperatura medio ambiente. 
Tabla 2. Porcentaje de viabilidad según la prueba de TZ. Separación de medias por la prueba de Tukey.

\begin{tabular}{ccccc}
\hline $\begin{array}{l}\text { Condiciones } \\
\text { de almacenamiento }\end{array}$ & Lote & $15 / 03 / 2001$ & $25 / 09 / 2001$ & Anova \\
\hline $11^{\circ} \mathrm{C}$ & 15 & $52,5 \mathbf{a}$ & $49,9 \mathbf{a}$ & $\mathrm{ns}$ \\
$11^{\circ} \mathrm{C}$ & 28 & $48,8 \mathbf{a}$ & $44,5 \mathbf{a}$ & $\mathrm{ns}$ \\
$11^{\circ} \mathrm{C}$ & 34 & $63,4 \mathbf{a}$ & $63,1 \mathbf{a}$ & $\mathrm{ns}$ \\
Medio ambiente & 41 & $48,9 \mathbf{a}$ & $52,5 \mathbf{a}$ & $\mathrm{ns}$ \\
Medio ambiente & 50 & $40,0 \mathbf{a}$ & $12,1 \mathbf{b}$ & $*$ \\
HDS & & 30.65 & 13.26 & \\
\hline
\end{tabular}

Separación de medias por el factor HDS de Tukey $(\mathrm{p}=0,05)$; entre lotes. Valores con la misma letra son estadísticamente iguales. Análisis de varianza (Anova) entre fechas (lado derecho) donde: ** p $<0,01 * \mathrm{p}<0,05$ $\mathrm{ns}=$ no significativa.

para ambas fechas se obtuvo la variación del porcentaje de viabilidad en el tiempo; hallándose una tendencia a disminuir, pero en el lote 50 la disminución es estadísticamente significativa. Nótese además que los lotes almacenados a medio ambiente tienen porcentajes de viabilidad menores a los almacenados a $11^{\circ} \mathrm{C}$ (Tabla 2 y Anexo 1).

\section{Evaluación del contenido de humedad}

El contenido de humedad de las semillas varió de 5,7 a 15\%. Los lotes almacenados al medio ambiente presentaron mayor contenido de humedad $(>10,5 \%)$ que los almacenados a $11^{\circ} \mathrm{C}(<10,2 \%)$ para ambas fechas (Tabla $3)$. Además se puede observar un incremento significativo del porcentaje de humedad de las semillas en el tiempo (Tabla 3).

Tabla 3. Variación del contenido de humedad de las semillas en peso seco de los lotes, para las fechas evaluadas. Estas estuvieron almacenadas en bolsas con cierre.

\begin{tabular}{lccc}
\hline Lote & $\mathbf{2 1 / 0 3 / 0 1}$ & $\mathbf{2 6 / 0 6 / 0 1}$ & Anova \\
\hline 15 & $6,03 \mathbf{b}$ & $7,47 \mathbf{b}$ & $*$ \\
28 & $5,72 \mathbf{b}$ & $7,84 \mathbf{b}$ & $*$ \\
34 & $5,71 \mathbf{b}$ & $7,94 \mathbf{b}$ & $*$ \\
41 & $11,0 \mathbf{a}$ & $14,77 \mathbf{a}$ & $* *$ \\
50 & $10,6 \mathbf{a}$ & $15,19 \mathbf{a}$ & $*$ \\
$H D S$ & 1,89 & 1,14 & \\
\hline
\end{tabular}

Separación de medias por el factor HDS de Tukey (p $=0,05$ ); entre lotes. Valores con la misma letra son estadísticamente iguales. Análisis de varianza (Anova) entre fechas (lado derecho) donde: ${ }^{* *} \mathrm{p}<0,01 ; * \mathrm{p}<0,05 ; \mathrm{ns}=$ no significativa

\section{Efecto de la luz}

Se obtuvo diferencias altamente significativas al comparar los tratamientos de luz y oscuridad para todos los lotes, donde los tratamientos de oscuridad presentan los menores valores de porcentaje de germinación $(\% \mathrm{G}$ $<6 \%$ ) y de índice de velocidad de germinación $(\mathrm{IVG}<0,02)$ tanto en condiciones naturales (CN 2) y condiciones controladas (CC 4). En comparación, los dos tratamientos de luz los superan $(\% \mathrm{G}>24 \%$ y IVG $>1,3)($ Tabla 4$)$.

Comparando el efecto de las condiciones de almacenamiento en lotes con tratamiento de luz (CN 1 y CC 3) se tiene diferencias altamente significativas (Tabla 4), donde los lotes almacenados a medio ambiente (lotes 41 y 50) tienen los menores valores de porcentaje de germinación (de $24 \%$ a $44 \%$ ) y de IVG (de 1,3 a 2,5 ) en comparación a las almacenadas a $11^{\circ} \mathrm{C}(\% \mathrm{G}$ de $35 \%$ a $85 \%$ y IVG de 1,6 a 4,8 ).

\section{Efecto de la temperatura}

Al comparar los tratamientos CN1 y CN5 de temperatura variable y $\mathrm{CC} 3$ de temperatura constante (Tabla 5) se tiene diferencias significativas en los tratamientos, donde CN1 y CC3 presentan los mayores valores de porcentaje de germinación e índice de velocidad de germinación que el tratamiento $\mathrm{CN} 5$ de temperatura variable y alta.

Comparando los lotes según las condiciones de almacenamiento (Tabla 5) se obtuvo que los lotes almacenados a $11^{\circ} \mathrm{C}$ tienen los 
Tabla 4. Efecto de la luz en el porcentaje de germinación (\%G) e índice de velocidad de germinación (IVG).

\begin{tabular}{lcccccccccc}
\hline & \multicolumn{2}{c}{ Luz } & \multicolumn{2}{c}{ Oscuridad } & \multicolumn{2}{c}{ Luz } & \multicolumn{2}{c}{ Oscuridad } & \multicolumn{2}{c}{ CC } \\
LN 1 & \multicolumn{2}{c}{ CN 2 } & \multicolumn{2}{c}{ CC 4 } & \multicolumn{2}{c}{ HDS } \\
Lote & \% G & IVG & \% G & IVG & \% G & IVG & \% G & IVG & $\% \mathrm{G}$ & IVG \\
\hline 15 & $70,3 \mathrm{aB}$ & $4,6 \mathrm{aB}$ & $0,0 \mathrm{aC}$ & $0,0 \mathrm{aC}$ & $81,4 \mathrm{aA}$ & $5,5 \mathrm{aA}$ & $2,3 \mathrm{aC}$ & $0,05 \mathrm{aC}$ & 7,80 & 0,462 \\
28 & $64,3 \mathrm{abA}$ & $4,1 \mathrm{aA}$ & $0,3 \mathrm{aB}$ & $0,01 \mathrm{aB}$ & $53,7 \mathrm{bA}$ & $3,5 \mathrm{bA}$ & $0,6 \mathrm{aB}$ & $0,03 \mathrm{aB}$ & 12,92 & 1,138 \\
34 & $74,9 \mathrm{aA}$ & $5,3 \mathrm{aA}$ & $0,3 \mathrm{aB}$ & $0,01 \mathrm{aB}$ & $84,9 \mathrm{aA}$ & $5,8 \mathrm{aA}$ & $1,4 \mathrm{aB}$ & $0,05 \mathrm{aB}$ & 10,66 & 1,048 \\
41 & $44,0 \mathrm{bcA}$ & $2,4 \mathrm{bA}$ & $0,6 \mathrm{aB}$ & $0,02 \mathrm{aB}$ & $39,4 \mathrm{bcA}$ & $2,0 \mathrm{cA}$ & $1,4 \mathrm{aB}$ & $0,03 \mathrm{aB}$ & 7,65 & 0,587 \\
50 & $24,6 \mathrm{cA}$ & $1,3 \mathrm{bA}$ & $0,3 \mathrm{aB}$ & $0,01 \mathrm{aB}$ & $24 \mathrm{cA}$ & $1,3 \mathrm{cA}$ & $2,3 \mathrm{aB}$ & $0,03 \mathrm{aB}$ & 7,11 & 0,444 \\
HDS & 12,27 & 1,32 & 4,61 & 0,045 & 11,78 & 0,99 & 9,079 & 0,098 & & \\
\hline
\end{tabular}

Separación de medias por el factor HDS de Tukey $(\mathrm{p}=0,05)$; entre lotes por tratamiento en la parte inferior (letras minúsculas) y entre tratamientos por lote al lado derecho (letras mayúsculas). Valores con la misma letra son estadísticamente iguales.

mejores valores de porcentaje de germinación y de índice de velocidad de germinación que los lotes 41 y 50 que provienen de almacenamiento a medio ambiente. La germinación de $P$. raimondii se inició a los nueve días en todos los tratamientos aplicados.

\section{Discusión}

\section{Viabilidad y características de la semilla}

Las semillas de Puya raimondii presentaron una amplia variedad de características, apariencia, color, contenido de humedad y viabilidad. Esto puede explicarse por las observaciones realizadas al momento de la colecta, las semillas procedieron de plantas que pudieron estar sometidas a quemas (durante o después de la floración), con frutos expuestos al picoteo de aves, al ataque de larvas y hongos.

Las semillas caracterizadas como blancas $\mathrm{y}$ buenas tuvieron los mayores valores de viabilidad y bajos contenidos de humedad. Al res- pecto Soplin (1988) y Besnier (1989) mencionaban que las condiciones de maduración, como la temperatura y humedad en que las semillas permanecen influyen en su viabilidad por lo tanto en su calidad.

En los lotes seleccionados para las pruebas de germinación, al correlacionar los resultados de la prueba de viabilidad con el porcentaje de humedad de las semillas (Pearson, $\mathrm{p}<0.01)$, se obtuvo una correlación inversa y significativa; donde las semillas con alto contenido de humedad tienen menos viabilidad. Las semillas almacenadas al medio ambiente ( $21^{\circ} \mathrm{C}$ aproximadamente) incrementaron su contenido de humedad (de 10,80 a 14,98\%) más que las de almacenadas a $11^{\circ} \mathrm{C}(\mathrm{de} 5,91$ a 8,35\%). De allí que los lotes almacenados a medio ambiente tuvieron menor viabilidad que los almacenados a $11^{\circ} \mathrm{C}$; encontrándose que estos factores (contenido de humedad y temperatura de almacenamiento) son importan-

Tabla 5. Efecto de la temperatura variable $\left(\mathrm{CN} 1: 18,5-23^{\circ} \mathrm{C}\right.$ y CN5: $21-26,5^{\circ} \mathrm{C}$ y constante $(\mathrm{CC} 3$ : $18^{\circ} \mathrm{C}$ ) en el porcentaje de germinación (\%G) e índice de velocidad de germinación (IVG).

\begin{tabular}{lcccccccc}
\hline & \multicolumn{2}{c}{ CN 1 } & \multicolumn{2}{c}{ CC 3 } & \multicolumn{2}{c}{ CN 5 } & \multicolumn{2}{c}{ HDS } \\
Lote & \% G & IVG & \% G & IVG & \% G & IVG & \%G & IVG \\
\hline 15 & $70,3 \mathrm{aA}$ & $4,6 \mathrm{aB}$ & $81,4 \mathrm{aA}$ & $5,5 \mathrm{aA}$ & $44,8 \mathrm{bB}$ & $0,7 \mathrm{bC}$ & 11,62 & 0,69 \\
28 & $64,3 \mathrm{abA}$ & $4,1 \mathrm{aA}$ & $53,7 \mathrm{bA}$ & $3,5 \mathrm{bA}$ & $20,9 \mathrm{bB}$ & $0,5 \mathrm{bB}$ & 15,52 & 1,35 \\
34 & $74,9 \mathrm{aA}$ & $5,3 \mathrm{aA}$ & $84,9 \mathrm{aA}$ & $5,8 \mathrm{aA}$ & $76 \mathrm{aA}$ & $1,6 \mathrm{aB}$ & 15,87 & 1,45 \\
41 & $44,0 \mathrm{bcA}$ & $1,4 \mathrm{bA}$ & $39,4 \mathrm{bcA}$ & $2,0 \mathrm{cA}$ & $19,9 \mathrm{bB}$ & $0,5 \mathrm{bB}$ & 8,06 & 0,71 \\
50 & $24,6 \mathrm{cA}$ & $1,3 \mathrm{bA}$ & $24,0 \mathrm{cA}$ & $1,3 \mathrm{cA}$ & $10,1 \mathrm{bB}$ & $0,2 \mathrm{bB}$ & 6,94 & 0,54 \\
HDS & 12,27 & 1,32 & 11,77 & 0,99 & 15,72 & 0,63 & & \\
\hline
\end{tabular}

Separación de medias por el factor HDS de Tukey $(\mathrm{p}=0,05)$; entre lotes por tratamiento en la parte inferior (letras minúsculas) y entre tratamientos por lote al lado derecho (letras mayúsculas). Valores con la misma letra son estadísticamente iguales. 
tes en el mantenimiento de la viabilidad de la semilla, como lo mencionan Roberts (1972), Soplin (1988) y Berrie (1990).

\section{Factores de luz y temperatura en la germinación}

Se encontró el efecto positivo de la luz en la germinación de las semillas de $P$. raimondii ya que los porcentajes de germinación e IVG de los tratamientos con luz, de todos los lotes, superaron a los tratamientos de oscuridad, corroborando los resultados obtenidos obtenidos por Suni et al. (2001); lo cual indica que las semillas de esta especie son fotoblásticas positivas según la clasificación que hace Berrie (1990). Esto también concuerda con lo dicho por Smith \& Downs (1974) y citado por Mercier \& Guerreiro (1990) quienes mencionan que las radiaciones cíclicas o luz continua promueven la germinación en muchas de las Bromelias, afectando la velocidad de germinación.

Así mismo se encontró que temperaturas al$\operatorname{tas}\left(>21{ }^{\circ} \mathrm{C}\right)$ afectan negativamente la germinación de las semillas de $P$. raimondii disminuyendo entre 10 y $40 \%$ (dependiendo del lote en los tratamientos evaluados), resultados semejantes se encuentra en Pitcairnia flamea que disminuye su porcentaje de germinación en 17\% cuando la temperatura aumenta de 15 a $25{ }^{\circ} \mathrm{C}$ (Mercier \& Guerreiro, 1990). Esto nos indica que temperaturas menores a $21^{\circ} \mathrm{C}$ serían más adecuadas para la germinación de $P$. raimondii.

Los valores de tiempo de germinación indicados por Rivera (1985) y De La Cruz (2000) diferentes al inicio de germinación obtenidos en el presente trabajo podrían deberse a criterio de evaluación de las semillas o a condiciones experimentales.

Asimismo el índice de correlación Pearson fue significativo $(p<0,01)$ entre el porcentaje de germinación y el porcentaje de viabilidad obtenidos con TZ; a pesar que los valores obtenidos para el porcentaje de germinación fueron mayores a los de TZ; el método del tetrazolium sería una manera rápida de estimar la viabilidad de las semillas como lo indica Delouche et al. (1971).

\section{Conclusiones}

La germinación de las semillas de $P$. raimondii es dependiente de la luz siendo fotoblásticas positivas, por lo tanto controladas por el fitocromo. Además las temperaturas altas $\left(>21{ }^{\circ} \mathrm{C}\right)$ disminuyen el porcentaje de germinación e IVG.

Las semillas de Puya raimondii presentan adaptaciones adecuadas para su dispersión: son numerosas, pequeñas (3 a $4 \mathrm{~mm})$, livianas $(0,48$ $\mathrm{mg} / \mathrm{semilla}$ ), aladas y con alto porcentaje de germinación; sin embargo los daños ocasionados por las aves, hongos y larvas de polilla en la fructificación disminuye el número de semillas viables y vigorosas; además los requerimiento de la semilla para su germinación (luz, humedad y temperatura) limitan los microhabitats que pueden colonizar; si a esto se agrega el efecto negativo del hombre, como la tala, quema, pastoreo y expansión de las zonas agrícolas que ocasionan una disminución de su población; se restringe aun más su posibilidad de establecimiento.

\section{Literatura citada}

Berrie, A. 1990. Germination and Dormancy. En: Malcom B. Wilking (ed.). Advanced Plant Physiology. USA. p. $440-468$

Cano, A., La Torre M. \& Ramírez A. 2000, Aspectos Cuantitativos Sobre La Biología De La Puya raimondii Harms (Bromeliaceae). Resumen del VIII Congreso Nacional de Botánica. p.12.

Delouche J., Wayne M., Raspet M.\& Lienhard M. 1971.Prueba de la viabilidad de la semilla con tetrazol. Mexico/buenos aires. 1ed.esp.71p.

Hartmann, O., 1981, Puya raimondii Cada Vez Son Menos. Boletín de Lima (10): 79-83

MalufA.M., 1998, Germinación Y Almacenamiento De Las Diasporas De Cerejeida (Eugenia involucrata D.C.- Myrtaceae) En Función Del Contenido De Agua. Revista Brasilera de semillas. 20: 184-188

Mercier H. \& O. Guerreiro 1990. Propagação Sexuada de Algunas Bromélias Nativas da Mata Atlántica: Efeito da Luz e da Temperatura na Germinação. Hoechnea 17(2):19-26

Rivera, C. A. 1985. Puya raimondii Harms. Boletín de Lima (38): 85-89

Roberts E. H. 1972. Viability of Seed. Chapman \& Hall Ltd. Great Britain. 446p.

Smith L. B. \& Downs, L. J. 1973. Flora Neotrópica (Pitcairnioideae, Bromeliaceae). Monograph $N^{\circ} 14$. 
Soplin H. 1988 Factores que afectan el potencial de almacenamiento de las semillas. Monografía «Conservación y uso de los recursos genéticos» INIA LIMA PERU

Steel, R.G.D. \& Torrie, J.H. 1980. Principles and Procedures of statistics. 2ed. N.Y. Mc Graw Hill. 481p.
Suni M., Cano A. \& Vadillo G. 2001 Ensayos preliminares en Puya raimondii Harms (BROMELIACEAE) Rev. Peruana de Biología. (8) 1: 53-59

Venero, J.L. \& Hostning R. 1986. Las «Titánicas» de Aymaraes (Apurímac). Boletín de Lima (48) 83-88

Anexo 1. Características de las semillas de Puya raimondii colectadas para el presente trabajo

\begin{tabular}{|c|c|c|c|c|c|c|c|c|}
\hline Lote & $\begin{array}{c}\text { Fecha de } \\
\text { colecta }\end{array}$ & $\begin{array}{c}\text { Fecha de } \\
\text { ingreso } \\
\text { al } \\
\text { labora. }\end{array}$ & $\begin{array}{l}\text { Posición } \\
\text { en } \\
\text { infrutes } \\
\text { cencia. }\end{array}$ & $\begin{array}{c}\text { Color de } \\
\text { las } \\
\text { semillas } \\
\end{array}$ & $\begin{array}{l}\text { Condición } \\
\text { aparente de } \\
\text { las semillas }\end{array}$ & $\begin{array}{c}\text { Condiciones } \\
\text { Almac.* }\end{array}$ & $\begin{array}{c}\text { Lotes de } \\
\text { semillas } \\
\text { (g) }\end{array}$ & $\begin{array}{l}\text { e } \\
\text { s } \\
\mathbf{N}^{\circ}\end{array}$ \\
\hline 1 & $15 / 12 / 99$ & $10 / 03 / 00$ & & blancas & buenas & Medio amb & - & 280 \\
\hline 2 & $15 / 12 / 99$ & $12 / 05 / 00$ & . & blancas & buenas & $11^{\circ} \mathrm{C}$ & 0,47 & 1000 \\
\hline 3 & $15 / 12 / 99$ & $15 / 09 / 00$ & & blancas & buenas & $11^{\circ} \mathrm{C}$ & 2,427 & 5000 \\
\hline 4 & $29 / 10 / 00$ & $02 / 11 / 00$ & apical & oscuras & buenas & $11^{\circ} \mathrm{C}$ & 0,85 & 1751 \\
\hline 5 & $29 / 10 / 00$ & $02 / 11 / 00$ & apical & oscuras & semiquemadas & $11^{\circ} \mathrm{C}$ & 0,07 & 144 \\
\hline 6 & $29 / 10 / 00$ & $02 / 11 / 00$ & apical & blancas & buenas & $11^{\circ} \mathrm{C}$ & 1,83 & 3770 \\
\hline 7 & $29 / 10 / 00$ & $02 / 11 / 00$ & apical & blancas & semiquemadas & $11^{\circ} \mathrm{C}$ & 0,07 & 144 \\
\hline 8 & $29 / 10 / 00$ & $02 / 11 / 00$ & medio & oscuras & semiquemadas & $11^{\circ} \mathrm{C}$ & 0,37 & 762 \\
\hline 9 & $29 / 10 / 00$ & 02/11/00 & medio & oscuras & quemadas & $11^{\circ} \mathrm{C}$ & 0,05 & 103 \\
\hline 10 & $29 / 10 / 00$ & $02 / 11 / 00$ & medio & blancas & buenas & $11^{\circ} \mathrm{C}$ & 0,04 & 82 \\
\hline 11 & $29 / 10 / 00$ & $02 / 11 / 00$ & basal & oscuras & buenas & $11^{\circ} \mathrm{C}$ & 5,78 & 11908 \\
\hline 12 & $29 / 10 / 00$ & $02 / 11 / 00$ & basal & oscuras & semiquemadas & $11^{\circ} \mathrm{C}$ & 2,09 & 4306 \\
\hline 13 & $29 / 10 / 00$ & $02 / 11 / 00$ & basal & oscuras & quemadas & $11^{\circ} \mathrm{C}$ & 0,718 & 1479 \\
\hline 14 & $29 / 10 / 00$ & $02 / 11 / 00$ & basal & oscuras & muy quemadas & $11^{\circ} \mathrm{C}$ & 0,28 & 577 \\
\hline 15 & $29 / 10 / 00$ & $02 / 11 / 00$ & basal & blancas & buenas & $11^{\circ} \mathrm{C}$ & 1,94 & 3997 \\
\hline 16 & $29 / 10 / 00$ & $02 / 11 / 00$ & basal & blancas & semiquemadas & $11^{\circ} \mathrm{C}$ & 0,5 & 1030 \\
\hline 17 & $29 / 10 / 00$ & $02 / 11 / 00$ & basal & blancas & quemadas & $11^{\circ} \mathrm{C}$ & 0,05 & 103 \\
\hline 18 & $29 / 10 / 00$ & $02 / 11 / 00$ & basal & rojas & buenas & $11^{\circ} \mathrm{C}$ & 0,16 & 330 \\
\hline 19 & $29 / 10 / 00$ & $02 / 11 / 00$ & basal & mixto & buenas & $11^{\circ} \mathrm{C}$ & 0,03 & 62 \\
\hline 20 & $29 / 10 / 00$ & $02 / 11 / 00$ & basal & mixto & semiquemadas & $11^{\circ} \mathrm{C}$ & 0,13 & 268 \\
\hline 21 & $29 / 10 / 00$ & $02 / 11 / 00$ & basal & especiales & buenas & $11^{\circ} \mathrm{C}$ & 0,01 & 21 \\
\hline 22 & $30 / 10 / 00$ & 02/11/00 & mixto & blancas & buenas & $11^{\circ} \mathrm{C}$ & 2,64 & 5439 \\
\hline 23 & $30 / 10 / 00$ & $06 / 11 / 00$ & mixto & blancas & buenas & $11^{\circ} \mathrm{C}$ & 57,431 & 118315 \\
\hline 24 & $30 / 10 / 00$ & $02 / 11 / 00$ & apical & blancas & semiquemadas & $11^{\circ} \mathrm{C}$ & 0,03 & 62 \\
\hline 25 & $30 / 10 / 00$ & $02 / 11 / 00$ & apical & oscuras & semiquemadas & $11^{\circ} \mathrm{C}$ & 0,05 & 103 \\
\hline 26 & $30 / 10 / 00$ & $02 / 11 / 00$ & apical & blancas & bu & $11^{\circ} \mathrm{C}$ & 0,16 & 330 \\
\hline 27 & $30 / 10 / 00$ & $02 / 11 / 00$ & medio & oscuras & semiquemadas & $11^{\circ} \mathrm{C}$ & 0,09 & 185 \\
\hline 28 & $30 / 10 / 00$ & 02/11/00 & medio & blancas & buenas & $11^{\circ} \mathrm{C}$ & 2,02 & 4162 \\
\hline 29 & $30 / 10 / 00$ & $02 / 11 / 00$ & medio & Rojas & semiquemadas & $11^{\circ} \mathrm{C}$ & 0,16 & 330 \\
\hline 30 & $30 / 10 / 00$ & $02 / 11 / 00$ & medio & mixto & buenas & ${ }^{\circ} \mathrm{C}$ & 0,21 & 433 \\
\hline 31 & $30 / 10 / 00$ & $02 / 11 / 00$ & basal & oscuras & quemadas & $11^{\circ} \mathrm{C}$ & 0,34 & 700 \\
\hline 32 & $30 / 10 / 00$ & $02 / 11 / 00$ & basal & oscuras & muy quemadas & $11^{\circ} \mathrm{C}$ & 0,08 & 165 \\
\hline 33 & $30 / 10 / 00$ & $02 / 11 / 00$ & basal & oscuras & semiquemadas & $11^{\circ} \mathrm{C}$ & 0,15 & 309 \\
\hline 34 & $30 / 10 / 00$ & $02 / 11 / 00$ & basal & blancas & buenas & $11^{\circ} \mathrm{C}$ & 3,83 & 7890 \\
\hline 35 & $30 / 10 / 00$ & $02 / 11 / 00$ & basal & rojas & buenas & $11^{\circ} \mathrm{C}$ & 0,42 & 865 \\
\hline 36 & $30 / 10 / 00$ & $02 / 11 / 00$ & basal & mixto & bue & $11^{\circ} \mathrm{C}$ & 0,47 & 968 \\
\hline 37 & $29 / 10 / 00$ & $06 / 11 / 00$ & & oscu & bu & dio am & 7,23 & 14895 \\
\hline 38 & $29 / 10 / 00$ & $06 / 11 / 00$ & mixto & oscuras & semiquemadas & dio amb. & 3,33 & 6860 \\
\hline 39 & $29 / 10 / 00$ & $06 / 11 / 00$ & mixto & oscuras & quemadas & medio amb. & 4,29 & 8838 \\
\hline 40 & $29 / 10 / 00$ & $06 / 11 / 00$ & mixto & oscuras & muy quemadas & medio amb. & 0,33 & 680 \\
\hline 41 & $29 / 10 / 00$ & 06/11/00 & mixto & blancas & buenas & medio amb. & 7,55 & 15554 \\
\hline 42 & $29 / 10 / 00$ & $06 / 11 / 00$ & mixto & blancas & semiquemadas & medio amb. & 3,69 & 7602 \\
\hline 43 & $29 / 10 / 00$ & $06 / 11 / 00$ & & rojas & & dis $a m b$ & 1,02 & 2101 \\
\hline 44 & $29 / 1$ & $06 / 1$ & mixto & rojas & semiquemadas & dio am & 0,35 & 721 \\
\hline 45 & $29 / 10 / 00$ & $06 / 11 / 00$ & & mixto & buenas & medio an & 0,66 & 1360 \\
\hline 46 & $29 / 10 / 00$ & $06 / 11 / 00$ & mixto & mixto & semiquemadas & medio amb. & 0,15 & 309 \\
\hline 47 & $29 / 10 / 00$ & $06 / 11 / 00$ & mixto & mixto & quemadas & medio amb. & 0,34 & 700 \\
\hline 48 & $29 / 10 / 00$ & 06/11/00 & mixto & especiales & regulares & medio amb. & 0,1 & 206 \\
\hline 49 & $30 / 10 / 00$ & $06 / 11 / 00$ & mixto & oscuras & buenas & medio amb. & 7,3 & 15039 \\
\hline 50 & $30 / 10 / 00$ & $06 / 11 / 00$ & mixto & blancas & & medio amb. & 4,83 & 9951 \\
\hline 51 & $30 / 10 / 00$ & $06 / 11 / 00$ & mixto & blancas & semiquemadas & medio amb. & 0,13 & 268 \\
\hline 52 & $30 / 10 / 00$ & $06 / 11 / 00$ & mixto & mixto & & dio an & 0,09 & 185 \\
\hline 53 & $30 / 10 / 00$ & $06 / 11 / 00$ & mixto & mixto & semiqu & medio amb. & 0,25 & 515 \\
\hline 54 & $30 / 10 / 00$ & $06 / 11 / 00$ & mixto & mixto & quemadas & medio amb. & 0,17 & 350 \\
\hline 55 & $30 / 10 / 00$ & $06 / 11 / 00$ & mixto & mixto & muy quemadas & medio amb. & 0,07 & 144 \\
\hline 56 & $30 / 10 / 00$ & $06 / 11 / 00$ & mixto & mixto & mixto & medio amb. & 6,81 & 14030 \\
\hline
\end{tabular}

